\title{
Size and temperature effects on dielectric breakdown of ferroelectric film capacitors
}

Sheng Tong ( $\square$ shengtg@hotmail.com )

Rapid Communication

Keywords: ceramics, Dielectric, energy density

Posted Date: September 17th, 2020

DOI: https://doi.org/10.21203/rs.3.rs-51187/v3

License: (c) (i) This work is licensed under a Creative Commons Attribution 4.0 International License. Read Full License 


\title{
Size and temperature effects on dielectric breakdown of ferroelectric films
}

Sheng Tong ${ }^{\mathrm{a}}$

DePaul University, 1 E. Jackson Blvd., Chicago, IL 60604, United States

\begin{abstract}
The paper introduces a model of dielectric breakdown strength. The model integrated thermal breakdown and defect models, representing the relationship between the electric field of ferroelectric films and dimensional parameters and operating temperature. This model is verified with experimental results of the lead lanthanum zirconate titanate (PLZT) films of various film thickness $(d=0.8-3 \mu \mathrm{m})$, electrode area $\left(A=0.0020-25 \mathrm{~mm}^{2}\right)$ tested under a range of operating temperature $(T=300-400 \mathrm{~K})$ with satisfying fitting results. Also learned is a relationship that the recoverable electric energy density is directly proportional to the square of breakdown electric field. This relationship is found viable in predicting the electric energy density in terms of variables of $d, A$, and $T$ for the PLZT films.
\end{abstract}

a) shengtg@gmail.com 
There has been a great interest in the dielectric breakdown (DBD) of the ferroelectric films for their important applications in passive devices, field emission transistors, and ferroelectric random-access memory (FeRAM). In these devices, DBD strength determines the degree of the integration and the magnitude of energy loss [1-3]. In particular, high performance energy storage capacitors require large DBD electric field $\left(E_{b}\right)$ and relative permittivity $\left(\varepsilon_{r}\right)$. The recoverable electric energy density $\left(U_{\text {reco }}\right)$ is calculated using the following equation [4]

$$
U_{\text {reco }}=\int_{E_{b}}^{0} \varepsilon_{0} \varepsilon_{r} E d E
$$

where $\varepsilon_{0}$ is the permittivity of vacuum. Early works on the mechanisms of DBD in high permittivity dielectrics (like ferroelectrics) found that the failure was not intrinsic in nature. That is, electronic breakdown featuring bond breaking and oxygen removal was not observed [5-8]. In this fashion, thermal breakdowns (TBD), such as dc TBD, impulse TBD, and avalanche breakdown, always dominate prior to intrinsic DBD. High-permittivity ferroelectric materials are characterized by their wide band-gap and electrical-to-thermal conductivity ratio $(\sigma / \kappa)$. Therefore, TBD is a commonly observed behavior in these materials including semiconductors and insulators [6]. Key factors dominating the breakdown strength of ferroelectric devices extrinsically include dielectric/electrode material properties (composition, microstructure), device structure (size and shape), and operational conditions (voltage ramp rate, temperature) [5,6,9-17]. There have been extensive research on the individual effect of dielectric thickness $(d)$, electrode area $(A)$, and temperature $(T)$ on the breakdown strength of typical ferroelectric materials such as $\mathrm{Pb}(\mathrm{Zr}, \mathrm{Ti}) \mathrm{O}_{3}$, $(\mathrm{Pb}, \mathrm{La})(\mathrm{Zr}, \mathrm{Ti}) \mathrm{O}_{3}(\mathrm{PLZT}), \mathrm{BaTiO}_{3},(\mathrm{Ba}, \mathrm{Sr}) \mathrm{TiO}_{3}$, etc [5,6,9-12,14-16]. However, a unified model on these parameters that affect DBD is yet to be developed. In this work, I developed a model on the correlation between $E_{b}$ and other key materials factors, such as dielectric thickness, area, and temperature. The validity of the model was tested by measurements of $E_{b}$ of PLZT films. 
Previous research suggested the dc TBD is the main mechanism, responsible for failure in high- $\varepsilon r$ dielectrics [5,6,16], although defect density was not considered. In these models, the dc electric field ramp rate is $\gg 1 \mu$ s and the relationship between $T$ and $E_{b}$ is as follows [6,15].

$$
-\kappa \nabla^{2} T=\sigma E_{b}^{2}
$$

It has also been reported that $E_{b}$ is inversely proportional to thickness $(d)$ which is mainly determined by the thermal distribution across the dielectric thickness and the bulk/surface thermal conductivity of the material [17-19]. Below a critical dielectric thickness, a power relationship between dielectric strength field $\left(E_{b}\right)$ and $d$ has been established: $[5,9,11]$

$E_{b}=c d^{-\alpha} \exp \left(E_{a} / 2 k T\right)$

where $c$ is a constant, $E_{a}$ is the activation energy, and $k$ is Boltzmann constant. The exponent $\alpha$ has a theoretical value of $0.5[11,17,18]$ while experimental values have been observed to vary between 0 and 1 depending on the processing conditions, thickness, etc. [5,7,9].

Although the TBD model in Eq. 3 provides the relationship between $d$ and $E_{b}$ of the ferroelectric films at large, the electrode area is not included. According to the thermal models $[5,6,16,19]$, heat generated during TBD can be effectively removed from the surface areas of the dielectric, leading to an increase in $E_{b}$. This is in contrast to the experimentally observed decrease in $E_{b}$ with increasing electrode area. However, this discrepancy can be explained by considering the probability of finding a more defective column with increase in area [12]. In practice, due to the defective nature of the capacitors, a number of samples are measured to statistically determine the reliable $E_{b}$ values. For example, one widely adopted method to determine average $E_{b}\left(E_{A}\right)$ is the Weibull distribution [20]. 
(a)

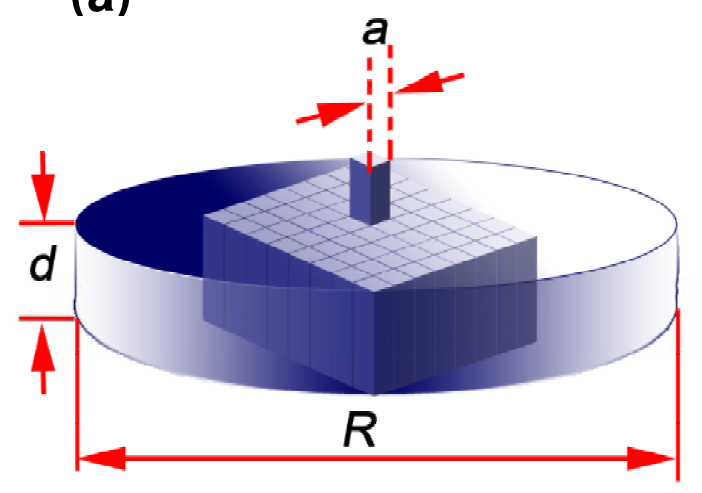

(b)

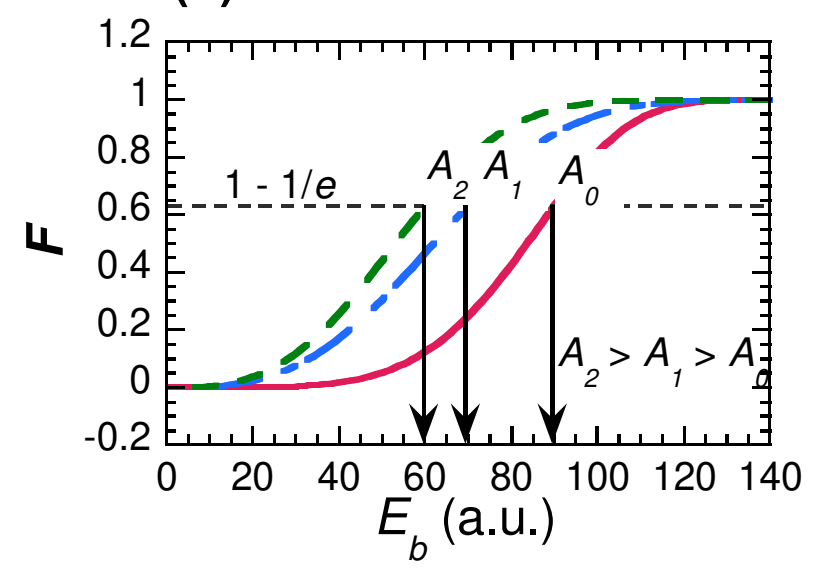

FIG. 1. (a) Illustration of a ferroelectric film with a thickness $(d)$ and diameter $(R)$ divided into small square columns with an edge length of $a$ for defect dielectric breakdown model analysis. (b) Weibull cumulative distribution function of the effect of electrode area on the average dielectric breakdown electric field $\left(E_{A}\right)$.

Defect models based on probability start with a small volume of defect randomly distributed in the bulk of the material. If the ferroelectric thin film is divided into a large number of small cubes with an edge length of $a$ representing the smallest defect size, the column (consisting of a stack of the cubes vertical to the surface) with the most defects can form a conducting path for the breakdown (Fig. 1a). This model is known as the weakest phase breakdown model [5,12,21,22]. In case of a larger electrode area, the probability of finding a conductive path under the electrode increases, leading to a reduction in $E_{b}$. As mentioned previously, the typical defective failure analysis is usually based on testing samples by varying several parameters $(T, d$, and $A)$ while keeping one of 
which a constant. In Weibull analysis, the cumulative probability $(F)$ corresponding to $E_{b}$ is expressed as follows [20],

$F(A)=1-e^{-\left(\frac{E_{b}}{E_{A}}\right)^{n}}$

where $F$ is the sample cumulative distributive parameter and $n$ is the fitting parameter. $E_{b}$ is equal to $E_{A}$ when $\mathrm{F}=1-1 / \mathrm{e}$. That is, at least one of such most defected unit columns is included under the electrode of area A. If a series of unit columns $\left(A_{0}=a^{2}\right)$ are tested, the values of $F$ as a function of $E_{b}$ can be illustrated as shown in Fig. 1b. When a series of electrode area of $A_{l}$ capacitors is tested, each column contains number of $\left(A_{l} / A_{0}\right)$ unit columns. Thus, to ensure $E_{b}$ to be greater than or equal to a certain value, each column must have less or equal number of defects defined by the value of $E_{b}$. That is, the relationship between the cumulative probability of $E_{b}$ for the electrode area of $A_{l}$ and that for the electrode area of $A_{0}$ can be expressed as follows

$$
1-F\left(A_{1}\right)=\left[1-F\left(A_{0}\right)\right]^{\frac{A_{1}}{A_{0}}}
$$

Using Eq. 4 and Eq. 5, we get

$E_{A_{1}}=E_{A_{0}}\left(\frac{A_{1}}{A_{0}}\right)^{-n^{-1}}$

$E_{A}$ of a finite electrode area is smaller than that of a unit column since $n$ is typically greater than that of an increasing failure rate [23]. As shown in Fig. 1b, the electrode area of $A_{l}$ greater than unit column leads to $E_{A_{1}}<E_{A_{0}}$, and a larger electrode area $A_{2}>A_{l}$ can result in a further reduced $E_{A}$. Assuming the BD strength of a unit column $E_{A 0}$ follows Eq. 2, it can be deduced that

$E_{A}=c d^{-\alpha} A^{-n} \exp \left(\frac{E_{a}}{2 k T}\right)$

where $c, \alpha, k$ are the fitting parameters, $E_{a}$ is the activation energy, and $k$ is the Boltzmann constant. Eq. 7 describes the interdependence of three variables, $d, A$, and $T$, that determine $E_{A}$ of the ferroelectric films. For any of the two variables being constant, the equation is reduced to Eq. 3 and 6, which has been widely discussed $[5,9,11,15]$. 


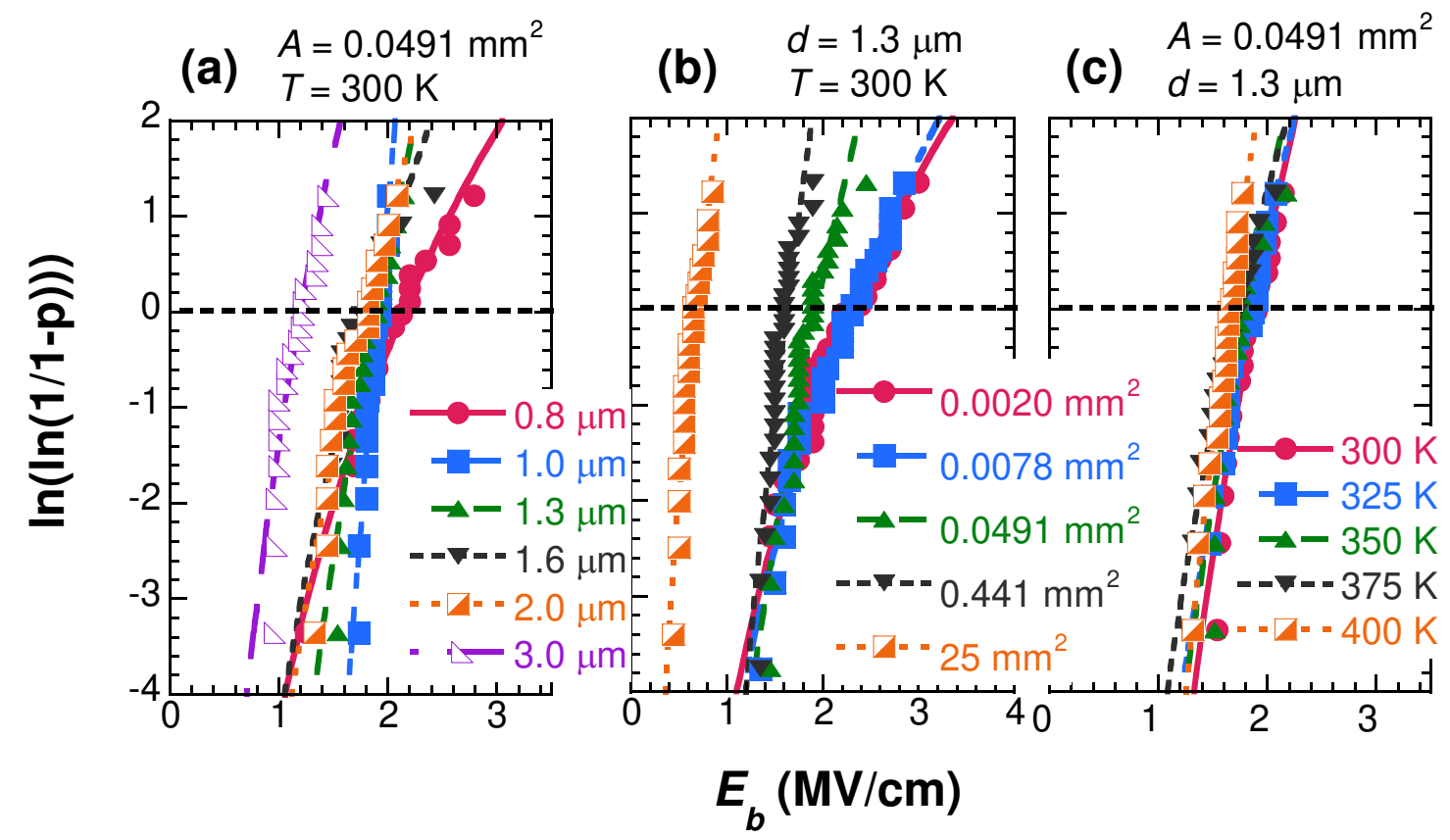

FIG. 2. Weibull failure estimation of dielectric breakdown electric field $\left(E_{b}\right)$ of PLZT films in different film thickness (a), electrode area (b), and under various temperature (c). $(\mathrm{R}>0.987)$

To validate Eq. 7, the lead lanthanum zirconate titanate $\left(\mathrm{Pb}_{0.92} \mathrm{La}\right.$.0.08 $\mathrm{Zr} 0.52 \mathrm{Ti}_{0.48} \mathrm{O}_{3}$, PLZT) films were deposited on the lanthanum nickel oxide buffered nickel substrates using chemical solution deposition with different thicknesses $(d \sim 0.8,1.0,1.3,1.6,2.0,3.0 \mu \mathrm{m})$, electrode sizes $(A \sim 0.0020$, $\left.0.0078,0.0491,0.441,25 \mathrm{~mm}^{2}\right)$, and measured the $E_{b}$ under different temperatures ( $T \sim 300,325$, $350,375,400 \mathrm{~K})$. The fabrication and measurements of PLZT films were reported previously [4]. For each condition, $E_{b}$ was measured on thirty samples for use in the Weibull analysis. Figs. 2ac show the Weibull statistical distribution of $E_{b}$ for various film thicknesses, electrode areas, and measurement temperatures. The experimental curves are fitted using Eqs. 4 and 8 [24]:

$$
F=(i-0.3) /(n+0.4)
$$

where $i$ is the sample sequence number and $n$ is the total number of sample $(\mathrm{n}=30)$. It can be seen in Figs. 2a-c, within the experiments conducted, electrode area exhibits the strongest effect on $E_{b}$, whereas $E_{b}$ is almost independent of temperature between 300 and $400 \mathrm{~K}$. The calculated $E_{A}$ 
from Fig $2 \mathrm{a}-\mathrm{c}$ is plotted in Fig. $3 \mathrm{a}-\mathrm{c}$ as a function of $d, A$, and $T$, with the error bars representing the measured $E_{b}$ range.
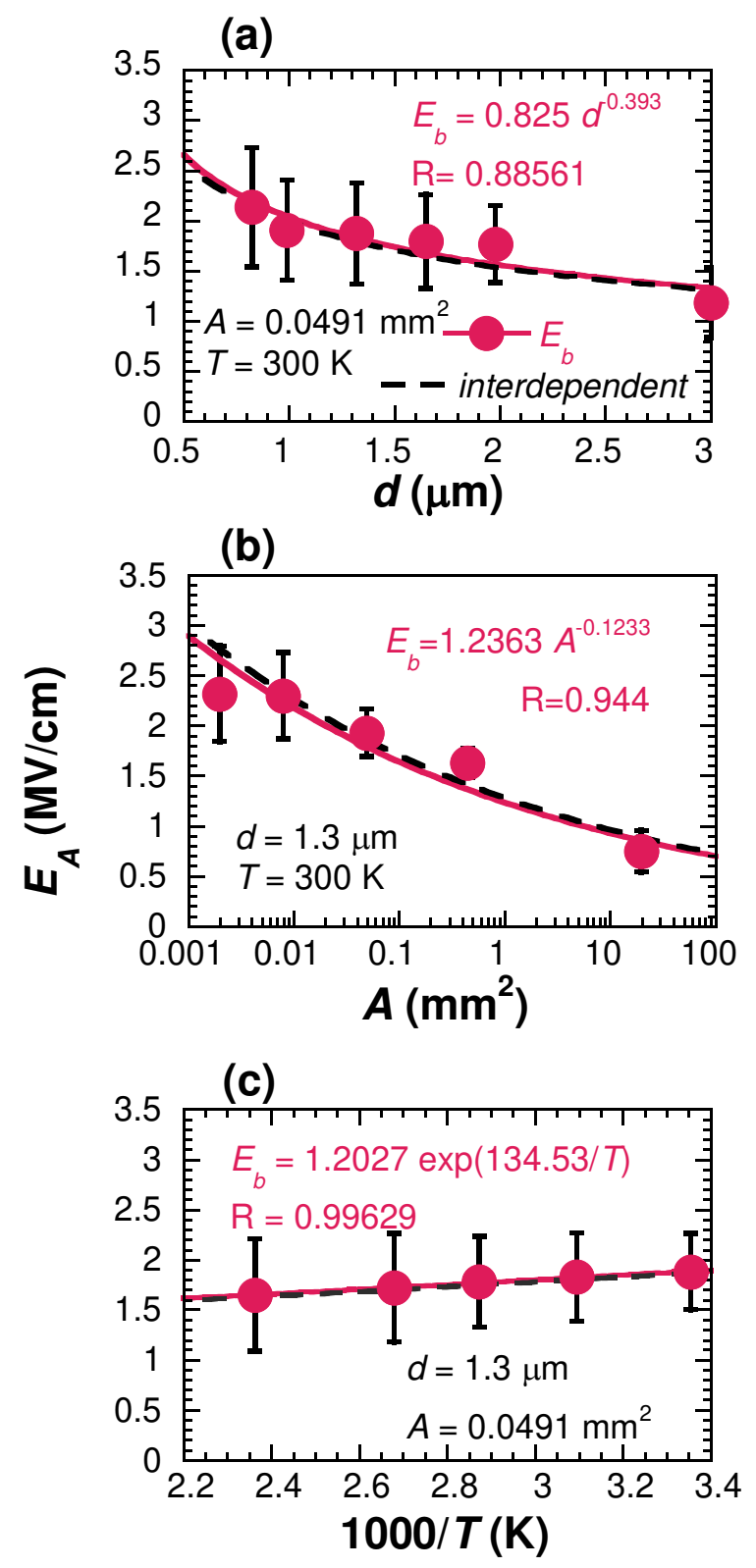

FIG. 3. Average dielectric breakdown electric field $\left(E_{A}\right)$ of PLZT films as function of film thickness (a), electrode area (b), and temperature (c). Solid red curves represent the fits with Eqs. 3 and 6; Broken black curves represent the fit using the unified Eq. 7. 
The effects of thickness, area, and temperature on $E_{A}$ are fitted using individual models based on Eq. 3 and 6 and represented as solid red lines in Fig. 3a - c. As can be seen in the figure, the individual equations effectively describe the dependence of $E_{A}$ on all three individual parameters with correlation coefficients between 0.88-0.99. It is observed that the of PLZT films follows the TBD model based on $d$ and $T$ similar to that observed by others $[10,11,13]$. The exponent ' $\alpha$,' calculated in Fig 3a, is $\sim 0.393$, which is close to that of reported for PZT [6,12].

The dependence of $E_{A}$ on $d, A$ and $T$ in Figs. $3 \mathrm{a}-\mathrm{c}$ are fitted with the unified equation (Eq. 7) including all three variables. The fits are in good agreement with the experimental values. The fitting parameters are: $c=0.88 \mathrm{MV} / \mathrm{cm}, \alpha=0.396, n=0.123, E_{a}=0.238 \mathrm{eV}$, and $R=0.867$. It clearly shows the interdependence of these variables $(d, A$, and $T)$ which play a vital role in the failure mechanism. Therefore, Eq. 7 can be used in failure analysis by predicting the $E_{b}$ values. The model can also be used for device optimization in specific applications. For example, the aspect ratio (diameter/thickness) of the thin film is essential to capacitors for energy storage application. For some energy storage properties such as $U_{\text {reco }}$, the corresponding polarization hysteresis loops were measured with the maximum electric field at $E_{A}$.

As shown in Fig. 4, the PLZT films exhibit polarization hysteresis loops with low coercive electric field $\left(E_{c}\right)$ and high saturation polarization $\left(P_{s}\right)$, typical of the relaxors which are prime candidates for energy storage applications $[25,26]$. Based on Eq. 1, high $U_{\text {reco }}$ is expected for materials with large $E_{b}$, for a given permittivity. $U_{\text {reco }}$ is calculated by integrating the area of the $P-E$ loop. The integrated recoverable energy density of the PLZT films as a function of $d, A$, and $T$ are plotted in Fig. 5. 

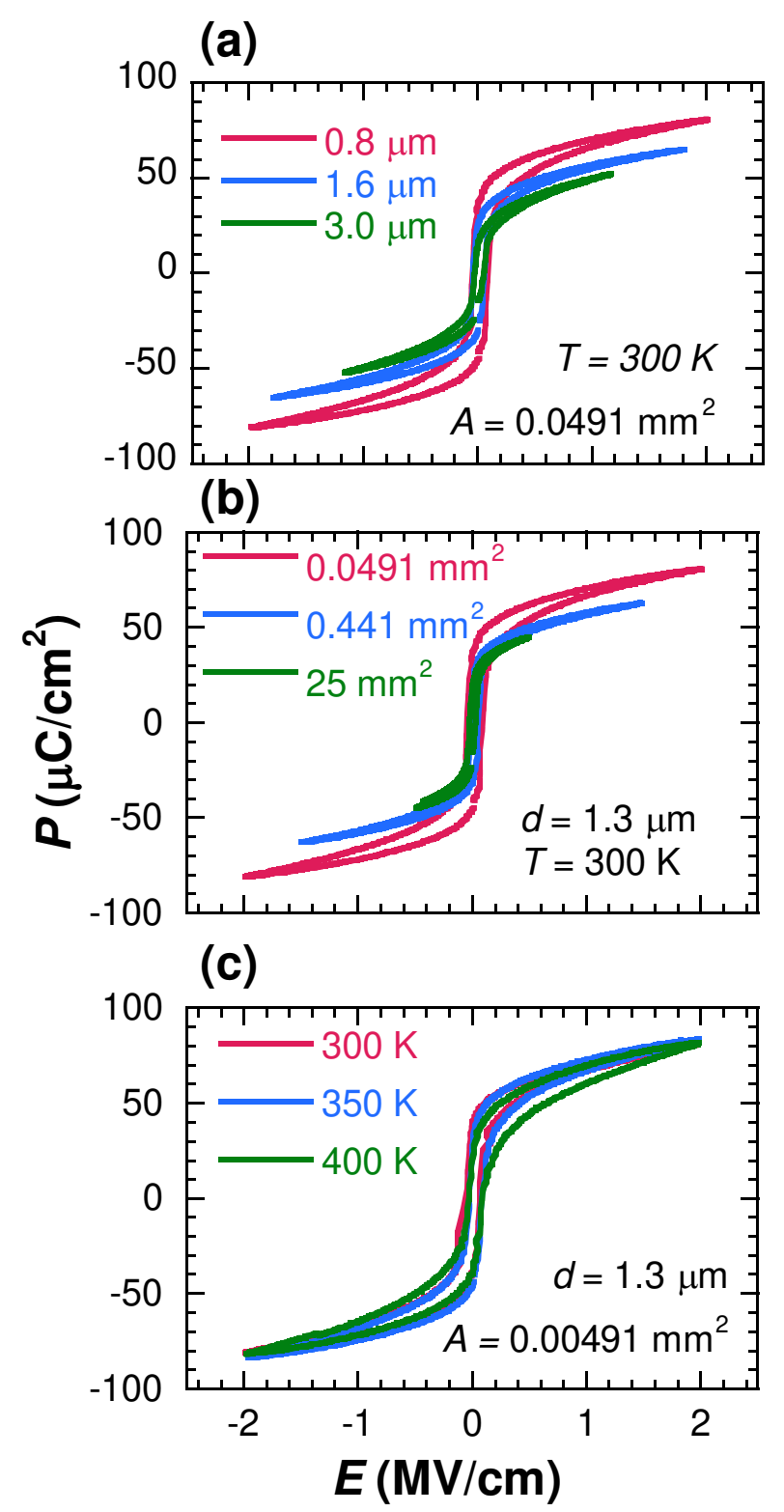

FIG. 4. Polarization hysteresis loops $\left(E_{\max }=E_{A}\right)$ of PLZT films as a function of film thickness (a), electrode area (b), and measurement temperature (c). 
(a)

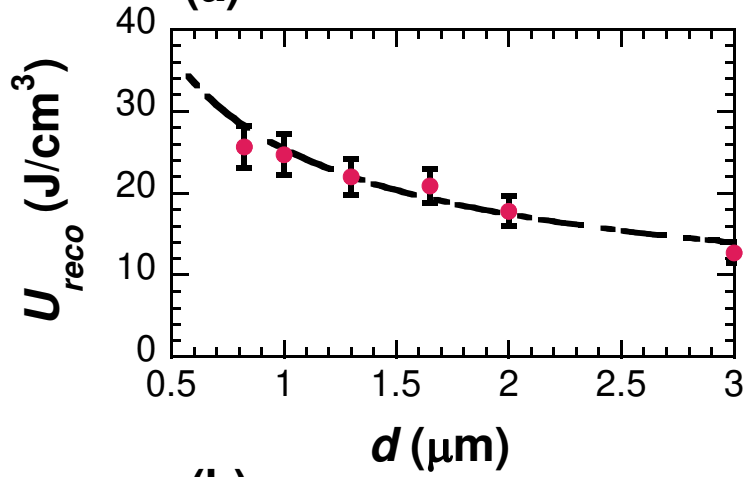

(b)

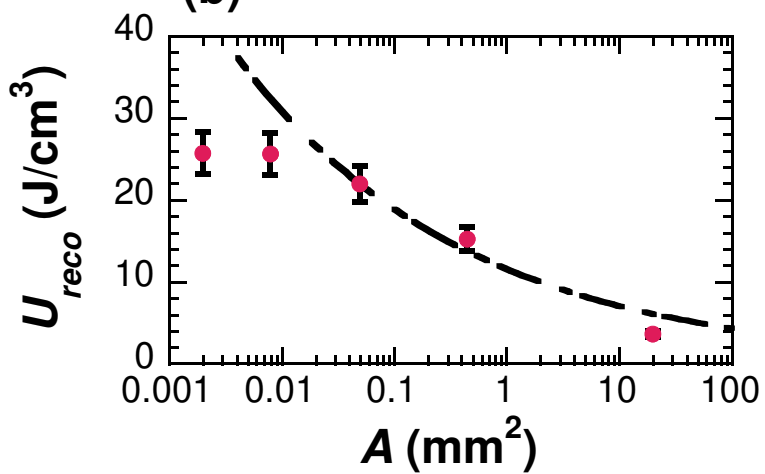

(c)

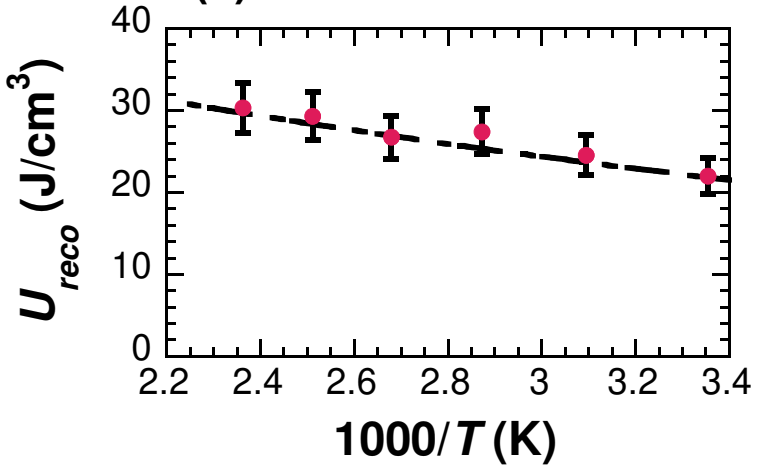

FIG. 5. Recoverable electric energy density $\left(U_{\text {reco }}\right)$ of PLZT films as function of film thickness (a), electrode area (b), and temperature (c).

Similar to $E_{b}, U_{\text {reco }}$ is also found to be strongly dependent on area and less so on temperature. Greater $U_{\text {reco }}$ is observed for samples with higher $E_{b}$ as shown in Fig. 5. For example, the value of $U_{\text {reco }}$ of the $0.8-\mu \mathrm{m}$-thick sample is as high as $\sim 26 \mathrm{~J} / \mathrm{cc}$ compared to $13 \mathrm{~J} / \mathrm{cc}$ for the $3-\mu \mathrm{m}$-thick film. Similar trend is observed with increasing area, as the $E_{b}$ decreases with increasing $d$ and $A$. Based on Eq. 1, we can write $U_{\text {reco }}=c d^{-\alpha} A^{-n} \exp \left(E_{a} / 2 k T\right)$ and fit the data in Fig 5. The fit (broken 
lines) is shown in Figs. $5 \mathrm{a}-\mathrm{c}$ and the parameters calculated are: $c=67.5 \mathrm{~J} / \mathrm{cc}, \alpha=0.544, n=$ $0.213, E_{a}=0.534 \mathrm{eV}$, and $R=0.768$. These fitting parameters of $\alpha, n, E_{a}$ are approximately twice of those obtained in Fig 3, indicating that $U_{\text {reco }}$ is proportional to the square of $E_{A}$. This implies that the polarization linearly increases as a function of the applied electric field at fields much greater than $E_{c}$. This square relationship agrees well with the fact that the PLZT is characteristically relaxor with nearly reversible $P-E$ loops $\left(E_{c} \sim 0.02 \mathrm{MV} / \mathrm{cm}\right)$.

Currently, the ferroelectric energy densities are typically reported with stressing their thicknesses rather than electrode areas. Given the equation above, it must be pointed out that a reduced electrode area, which is widely adopted in measuring ferroelectric thin films, could also play a role in its higher energy density than that of thicker films.

In conclusion, a unified equation has been developed on the relationship between the $E_{b}$ and related parameters such as the film thickness, electrode area, and temperature. The model has been found to agree well with the experimental results of the PLZT thin films in terms of all variables. Theoretical fits to the experimental data indicate the interdependence of the processing parameters. Polarization hysteresis loops of PLZT films are also obtained for predicting $U_{\text {reco }}$ based on the

developed model. A unique relationship of $U_{\text {reco }} \propto E_{A}^{2}$ is found, that is particularly useful in predicting the electric energy density in terms of variables $d, A$, and $T$.

\section{References}

1. Scott JF and Paz de Araujo CA, Science 246, 1400 (1989).

2. Setter N, Damjanovic D, Eng L, et al., J. Appl. Phys. 100, 051606 (2006).

3. Gregg JM, Phys. Status Solidi A 206, 577 (2009).

4. Tong S. Dielectric and Ferroelectric Properties of Lead Lanthanum Zirconate Titanate Thin Films for Capacitive Energy Storage. Ph.D. Thesis. Cincinnati (U.S.): University of Cincinnati. 
5. Scott JF, Annu. Rev. Mater. Sci. 28, 79 (1998).

6. J. Scott JF, Melnick BM, McMillan LD, et al., Integr. Ferroelectr. 3, 225 (1993).

7. Lee BH, Kang C, Choi R, et al., Appl. Phys. Lett. 94, 162904 (2009).

8. Li X, Tung C and Pey K, Appl. Phys. Lett. 93, 072903 (2008).

9. Agarwal VK and Srivastava VK, Thin Solid Films 8, 377 (1971).

10. Chen G, Zhao J, Li S, et al., Appl. Phys. Lett. 100, 222904 (2012).

11. Forlani F and Minnaja N, Phys. Stat. Sol. 4, 311 (1964).

12. Gerson R and Marshall TC, J. Appl. Phys. 30, 1650 (1959).

13. Lysne PC, J. Appl. Phys. 46, 230 (1975).

14. Ma B, Narayanan M and Balachandran U, Mater. Lett. 63, 1353 (2009).

15. Milliken AD, Bell AJ and Scott JF, Appl. Phys. Lett. 90, 112910 (2007).

16. Scott JF, Azuma M, De Araujo CA, et al., Integr. Ferroelectr. 4, 61 (1994).

17. Yoshimura M and Bowen HK, J. Am. Ceram. Soc 64, 404 (1981).

18. O'Dwyer JJ, IEEE Trans. Electr. Insul. EI-17, 484 (1982).

19. Moon PH, Instit. Electr. Engi. Trans. 50, 1008 (1931).

20. Dissado L, Fothergill J, Wolfe S, et al., Electric. Insulated. IEEE Transac. EI-19, 227 (1984).

21. Sune J, IEEE Electr. Dev. Lett. 22, 296 (2001).

22. Kimura M, IEEE Inter. Rel. Phys. Sym. 35, 190 (1997).

23. Mudholkar GS and Kollia GD, Commun. statist. theory methods 23, 1149 (1994). 
24. Dissado LA, J. Phys. D 23, 1582 (1990).

25. Cross LE, in Piezoelectricity, edited by Walter Heywang, Karl Lubitz and Wolfram Wersing (Springer, Berlin Heidelberg, 2008), Vol. 114, pp. 131-155.

26. Yamashita Y, Harada K and Saitoh S, Ferroelectrics 219, 29 (1998). 
Figures

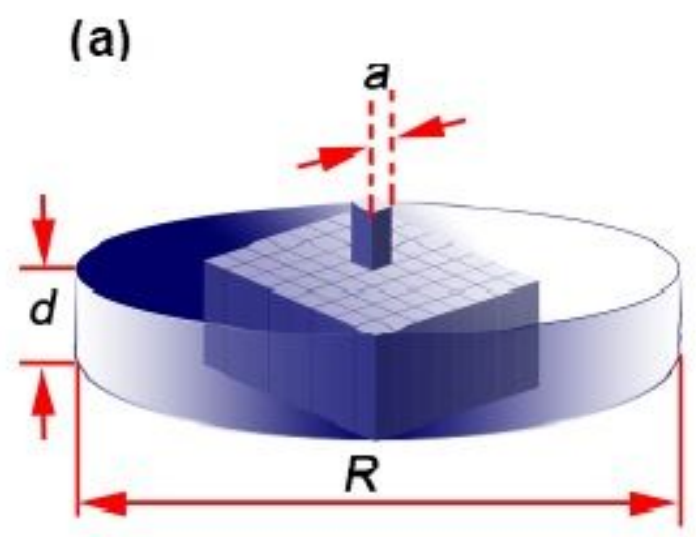

\section{(b)}

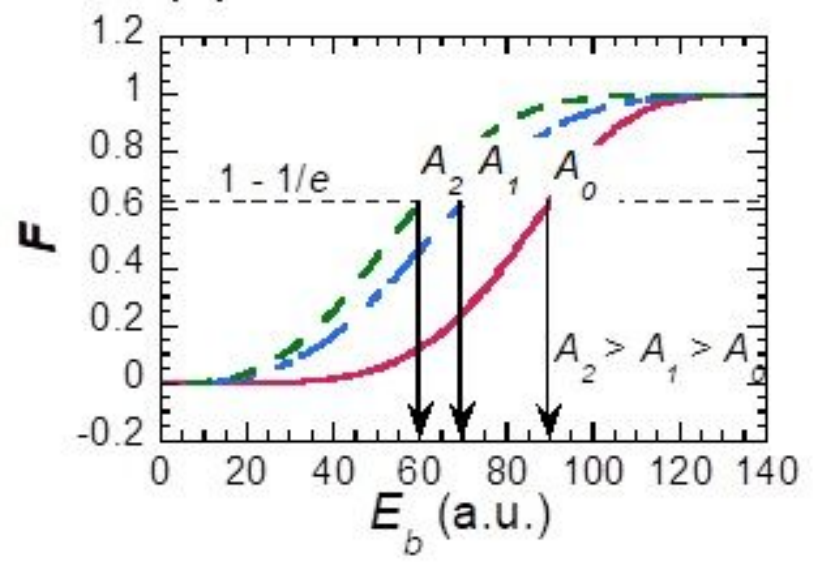

\section{Figure 1}

(a) Illustration of a ferroelectric film with a thickness (d) and diameter (R) divided into small square columns with an edge length of a for defect dielectric breakdown model analysis. (b) Weibull cumulative distribution function of the effect of electrode area on the average dielectric breakdown electric field (EA). 


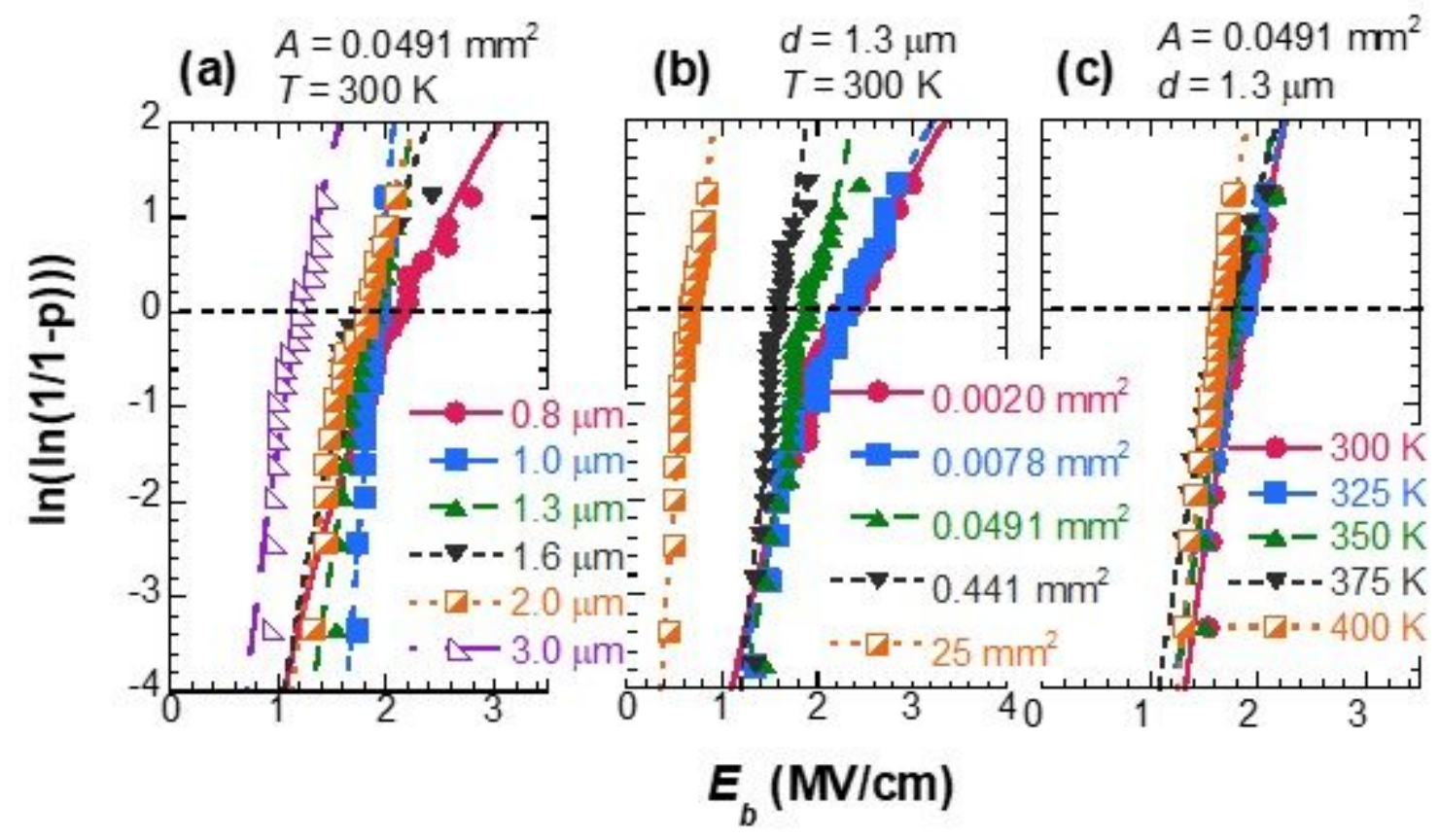

Figure 2

Weibull failure estimation of dielectric breakdown electric field (Eb) of PLZT films in different film thickness (a), electrode area (b), and under various temperature $(c)$. $(R>0.987)$ 

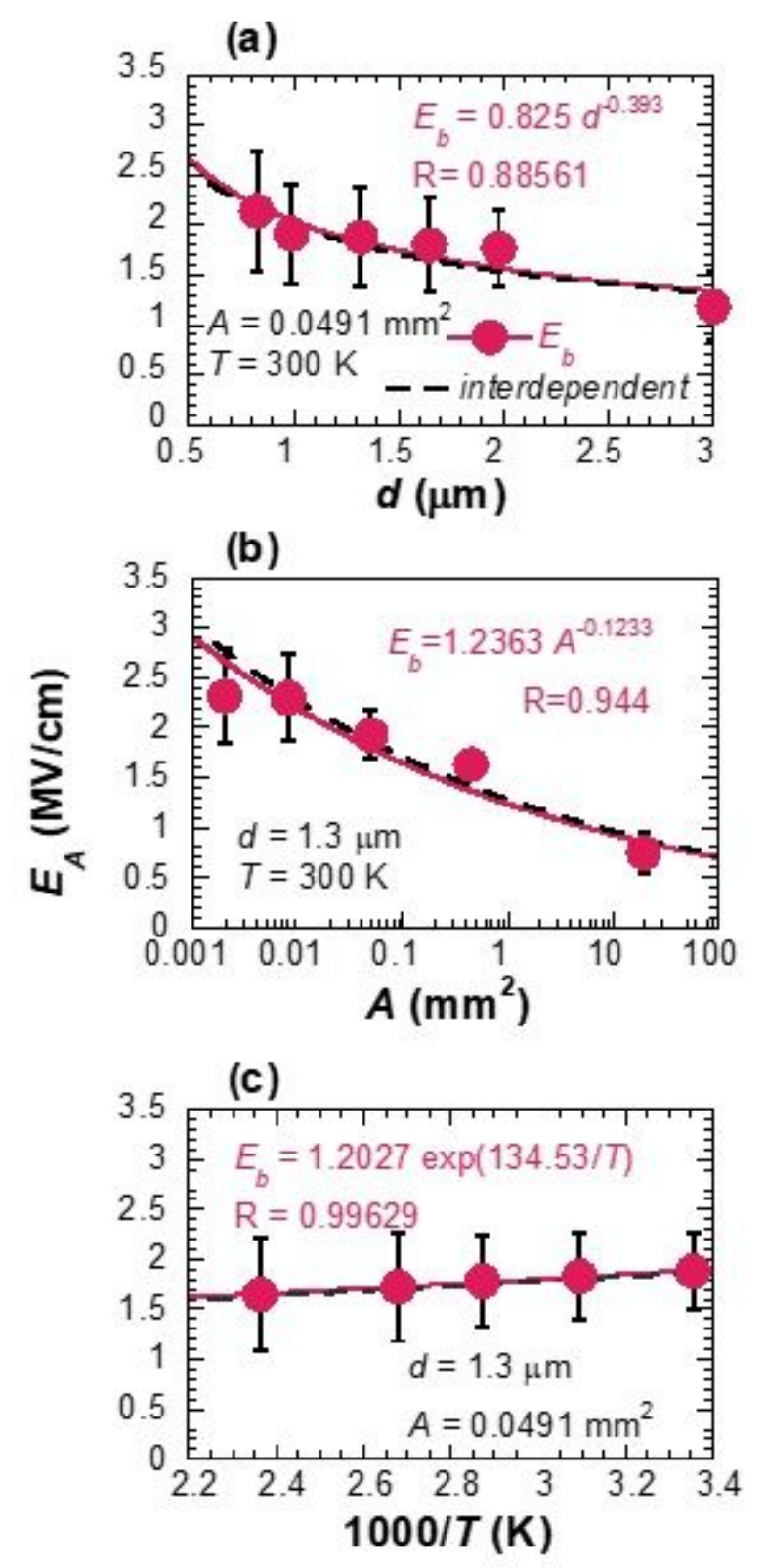

Figure 3

Average dielectric breakdown electric field (EA) of PLZT films as function of film thickness (a), electrode area (b), and temperature (c). Solid red curves represent the fits with Eqs. 3 and 6; Broken black curves represent the fit using the unified Eq. 7. 
(a)
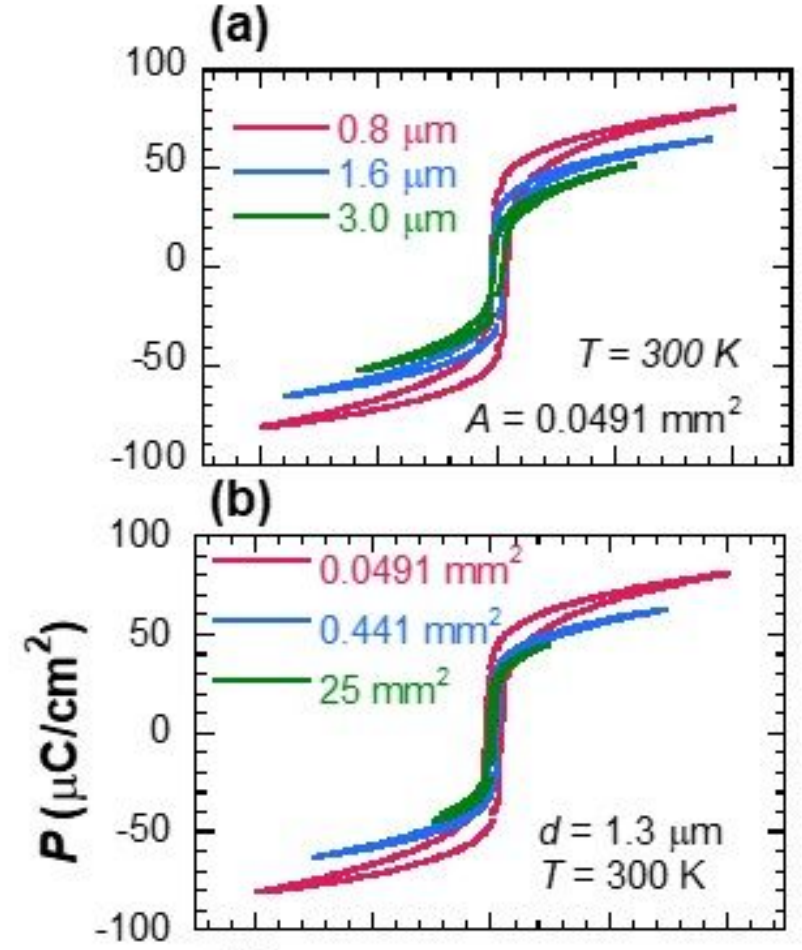

(c)

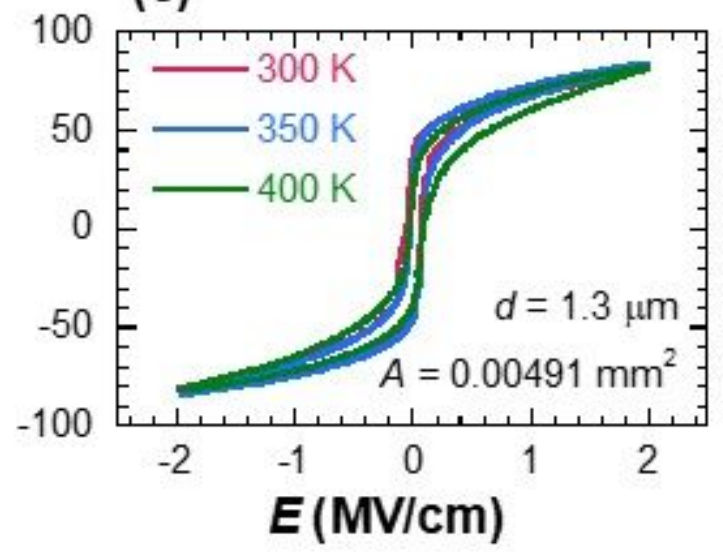

Figure 4

Polarization hysteresis loops (Emax = EA) of PLZT films as a function of film thickness (a), electrode area (b), and measurement temperature (c). 


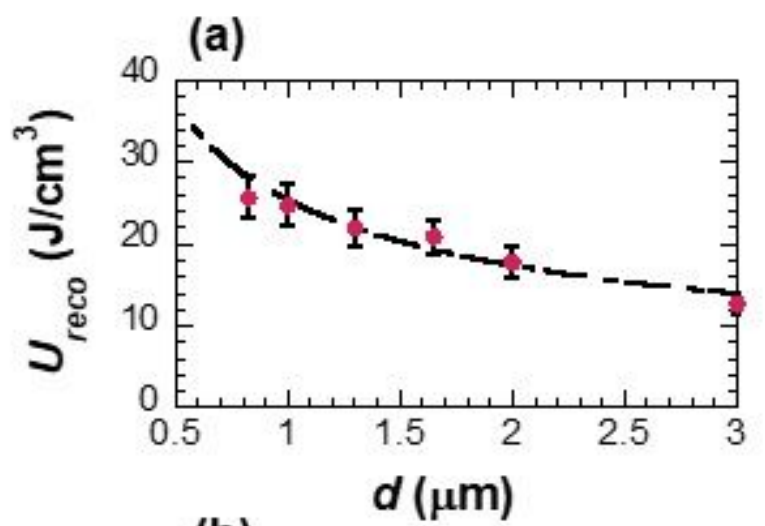

(b)

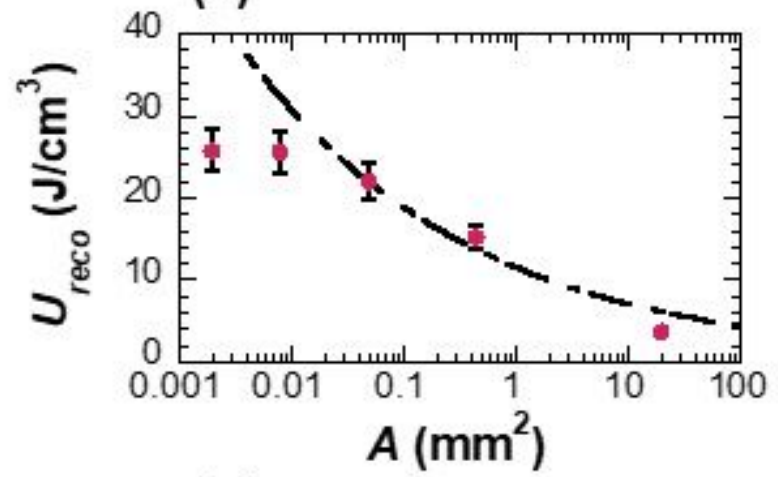

(c)

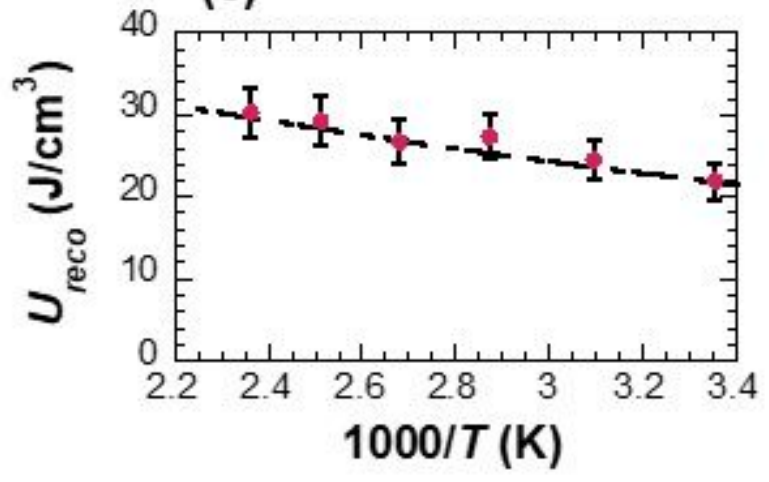

Figure 5

Recoverable electric energy density (Ureco) of PLZT films as function of film thickness (a), electrode area (b), and temperature (c). 